\title{
XVI. Ueber einige neue Mineralvorkommen des bayerischen Waldes.
}

\author{
Von \\ E. Weinschenk in Munchen.
}

(Mil 1 Textfigur.)

In den letzten Jahren wurden einige neue Mineralvorkommnisse in der Umgegend von B ode $\mathrm{n} \mathbf{m}$ a is und $\mathrm{Z}$ wie sel aufgefunden, von welchen vor allem diejenigen aus der Magnetkieslagerstätle erböhtes Interesse in Anspruch nehmen, da sie einestheils auf die genetischen Verhältnisse des Erzlagers Schlusse zu ziehen gestatten, anderntheils namentlich eines derselben als mineralogisch besonders beachtenswerth sich darstellte. Die neuen Mineralvorkommnisse, welche die an sich schon zahlreiche Reihe von mineralogisch interessanten Vorkommnissen der Bodenmais-Zwieseler Magnetkieslagerstätte um einige wichtige Glieder bereichern, wurden durch die Liberalität der königl. General-Bergwerks- und Salinen-Administration der hiesigen $S t a$ ats sammlung ubergeben; es sind Andalusit, sowie ein neues Vorkommen von Granat vom Silberberg bei Bodenmais und Turmalin von den neuerdings eröffneten Stollen am "Rolben Koth " bei Zwiesel.

Der Andalusit besitzt im bayerischen Walde eine weite Verbreitung einestheils als charakteristischer Gemengtheil zahlreicher Pegmatitgänge, sodann in Quarzlinsen in Gneiss und Glimmerschiefer und endlich als sicher constatirbare Contactbildung. Während aber die meisten dieser Vorkommnisse weilgehenden Umwandlungen unterlegen sind, welche zum Theil bis zur vollständigen Zersetzung des Minerals zu glimmerartigen Aggregaten fuhrten, lässt das neue, im Erzlager selbst aufgefundene Vorkommen keine Spur einer Zersetzung erkennen. Dasselbe wurde in neuen Anbruchen des Jahres 1893 entdeckt und zeigt den Andalusit in einzelnen langprismatischen Krystallen eingewachsen im Magnetkies, sowie in grossen Quarzkrystallen, welche selbst wieder von Magnetkies umhullt werden. Die mit 
diesen zusammen vorkommenden Mineralien, grüner Orthoklas und dunkelbrauner Biotit, führen keine Einschlissse von Andalusit. Wo die Individuen des letzteren Minerals im Magnetkies liegen, besitzen sie, wie die übrigen Krystalle von Silicaten, welche in demselben vorkommen, wie Cordierit, Oligoklas, Hypersthen etc., eine dunkle, fast schwarze Farbe, welche zum Theil von einem schwarzen, dünnen Ueberzuge herrthrt, zum Theil aber nach Entfernung des letzteren noch vorhanden ist und von einer grossen Menge von Einschlüssen von Magnetkies slammt, welche die mikroskopische Beobachtung in den meisten Krystallen erkennen lässt. Wo dagegen, was verhältnissmässig selten ist, das Mineral in Quarz eingewachsen ist, erscheint es vollkommen klar durchsichtig, von grunlicher bis röthlicher Farbe, und lässt den charakteristischen Pleochroismus des Andalusits schon dem blossem Auge deutlich erscheinen. Die einzelnen Krystalle sind $1-3 \mathrm{~cm}$ lang und selten uber $1 \frac{1}{2} \mathrm{~mm}$ dick, nur an einer Stelle findet sich eine divergentstrahlige Partie, welche etwas über $\frac{1}{2} \mathrm{~cm}$ Durchmesser besitzt.

Fur krystallographische Messungen sind die Krystalle trotz ihrer Frische nur wenig geeignet, da ausser einzelnen Flächen der Prismenzone nur noch \{101\} deutliche Reflexe gab; die übrigen Flächen konnten nur durch Schimmereinstellung bestimmt werden. Stets beobachtet man an den Krystallen in der Zone der Prismen $\{110\}$ und $\{210\}$ oft alternirend ausgebildet, und sehr untergeordnet die beiden verticalen Pinakoide; als Endausbildung treten fast immer $\{011\}$ und $\{101\}$ auf, an einigen Krystallen auch vereinzelte Flächen einer Form, welche der Pyramide $\{121\}$ nabesteht. Zu optischen Untersuchungen wurden einige der in Magnetkies eingewachsenen dunkeln Krystalle längere Zeit mit $\mathrm{HNO}_{3}$ digerirt, wodurch es gelang, die Einschlusse von Magnetkies herauszulösen; die Krystalle erscheinen jetzt röthlich und zeigen die normale optische Orienlirung, sowie den kräftigen Pleochroismus des Andalusits. $c=\mathfrak{a}$ intensiv blutroth, $b=\mathfrak{b}$ citrongelb mit Stich in's Zeisiggrine, $a=\mathfrak{c}$ licht weingelb. Absorption: $\mathfrak{a}>\mathfrak{b}>\mathfrak{c}$. Zu specifischen Gewichtsbestimmungen geeignetes Material konnte in Folge der zahlreichen Einschlusse nicht gewonnen werden, dagegen erschien das Mineral wegen seiner Frische zu Härtebestimmungen sehr geeignet. Es ergab sich, das Quarz unschwer geritzt wird, während Topas unangegriffen bleibt, die Härte des Minerals ist somit $7 \frac{1}{2}$.

Das zweite der neuen Mineralvorkommen stellt ein Granatmineral dar, welches sich schon bei blosser Betrachtung von dem normalen Granat der Lagerstätte ausser durch seine gelblichrothe Farbe, auch durch die Art seines Auftretens unterscheidet. Dasselbe stammt aus dem Bau „Gottesgabe Tiefstes « im Silberberg bei Bodenmais und zeigt den Granat in Krusten zusammengehäuft als Auskleidung von Klüften im Cordieritgneiss. Die verhältnissmässig grossen, bis $1 \mathrm{~cm}$ im Durchmesser aufweisenden Granatkrystalle silzen auf derben Aggregaten desselben Minerals auf und zeigen 
gegen die Mitle der Kluft zu wohlausgebildete Krystallformen mit glänzenden Flächen. Der Innenraum der Kluft wird von einem derben Gemenge von Magnetkies, Pyrit und Zinkblende eingenommen, welche aber beim Zerschlagen mit Leichtigkeit von den Granatkrystallen abspringen; von diesen Kluftausfüllungen strahlen oft schmale Adern derselben Zusammensetzung, meist aus vorherrschendem Granat bestehend, in den umgebenden Cordieritgneiss aus.

Was die Form betrifft, so sind die Krystalle zum Theil reine Dodekaëder, einige zeigen Abstumpfung der Kanten durch $\{211\}$, welches meist gestreift ist, wăhrend die Flächen von $\{110\}$ stets ungestreift sind, sowie durch vereinzelte schiefe, rauhe Flächen, welche auf Hexakisoktaëder mit complicirten Indices hinweisen, von deren genauer Identificirung aber wegen der Flächenbeschaffenheit abgesehen werden musste, zumal die Art des Auftretens dieser Flächen dieselben nicht als eigentliche Krystallfächen erscheinen lässt. Die grösseren Krystalle zeigen stets eine Anzahl von annähernd parallel, aber meist zackig verlaufenden Knickungen, welche auf eine Zertrummerung derselben durch secundäre, mechanische Einflusse hinweisen, womit auch die ganz ausserordentliche Sprödigkeit des Minerals zusammenhängt.

Da die Art des Vorkommens ebenso wie die Farbe, welche der des Hessonits am nächsten steht, auf eine von dem normalen Almandin des Gneisses abweichende Zusammensetzung schliessen liess, wurde das Mineral einer qualitativen Prüfung unterzogen, welche einen sehr hohen Gehalt an Mangan ergab, weshalb ich eine quantitative Analyse ausfuhrte. Das zu dieser verwendete Material bestand aus möglichst gereinigten Krystallen unter Ausschluss der derben Partien, auf welche dieselben aufgewachsen sind. Es blieb denselben aber immer noch ein geringer Rest von Pyrit beigemengt, welcher mechanisch nicht entfernt werden konnte, der aber das allgemeine Resultat der Analyse nur wenig beeinflussen konnte. Die Analyse wurde in der Weise ausgefuhrtt, dass das Mineral nach der gewöhnlichen Methode mit Kaliumnatriumearbonat aufgeschlossen und zunächst die Kieselsäure durch Eindampfen und Trocknen bei $120^{\circ}$ zur Abscheidung gebracht wurde. Da bei dem Ablösen fest anhaftender Reste der stark manganhaltigen Schmelze von den Wandungen des Platintiegels mittelst heisser $\mathrm{HCl}$ Spuren von Platin mit in Lösung gegangen waren, wurde sowohl die geglühte Kieselsäure mit Flusssäure entfernt und der Rückstand zurückgewogen, als auch in dem Filtrat durch Behandeln mit einer heiss concentrirten Lösung von Chlorammonium die geringe Menge Platin niedergeschlagen und entfernt. Hierauf wurden Eisenoxyd und Thonerde durch zweimal wiederholte Fällung mit Ammoniak vom Mangan getrennt, zusammen geglitht und gewogen. Ein Theil des gegluhten Gemenges dieser Oxyde wurde dann pulverisirt, mit $\mathrm{HKSO}_{4}$ aufgeschlossen und durch Behandeln mit 
reinster Kalilauge zerlegt. Endlich wurde die Spur Kalk als Oxalat gefällt und das Mangan als Pyrophosphat bestimmt. Die Niederschläge sowobl als die Filtrate wurden in jedem Falle auf ihre Reinheit gepruft, es ergab sich, dass die Trennungen und Fällungen vollständig quantitativ waren. Das Resultat dieser Analyse ist:

Angew. Substanz 0,5763 g.

\begin{tabular}{lc}
$\mathrm{SiO}_{2}$ & 34,65 \\
$\mathrm{Al}_{2} \mathrm{O}_{3}$ & 20,00 \\
$\mathrm{Fe}_{2} \mathrm{O}_{3}$ & 12,72 \\
$\mathrm{MnO}$ & 33,87 \\
$\mathrm{CaO}$ & Spur \\
$\mathrm{MgO}$ & 0,00 \\
\cline { 2 - 2 } & 101,24
\end{tabular}

Eine zur Bestimmung des Eisenoxydulgehaltes des Minerals nothwendige Menge war, ohne die wenigen, vorhandenen Stucke zu schädigen, nicht zu gewinnen; indess durfte die Granatformel als hinreichend festgestellt angesehen werden können, um eine Berechnung des Eisenoxydulgehaltes aus obiger Analyse zu gestatten. Wenn man das Mineral als eine isomorphe Mischung des vorherrschenden Manganoxydulthongranats mit untergeordnetem Eisenoxydulthongranat und sehr wenig Manganoxyduleisenoxydgranat ansieht, so ergiebt sich durch Umrechnung der nöthigen Menge Eisenoxyd in Oxydul eine Verminderung der Summe um genau 1\%; die Zusammensetzung dieses Granats ist daher :

\begin{tabular}{lr}
$\mathrm{SiO}_{2}$ & 34,65 \\
$\mathrm{Al}_{2} \mathrm{O}_{3}$ & 20,00 \\
$\mathrm{Fe}_{2} \mathrm{O}_{3}$ & 2,55 \\
$\mathrm{FeO}$ & 9,17 \\
$\mathrm{MnO}$ & 33,87 \\
\cline { 2 - 2 } & $\mathbf{1 0 0 , 2 4}$
\end{tabular}

Es liegt somit ein Spessartin vor, in welchem etwa $\frac{1}{5}$ des Manganoxyduls durch Eisenoxydul ersetzt ist.

Besonders interessant sind die optischen Verhältnisse dieses Vorkommnisses; es konnte allerdings nur eine \| (111) geschliffene Platte studirt werden, welche aus einem rein dodekaëdrisch ausgebildeten Krystalle etwa in der Mitte zwischen dem Centrum des Krystalles und seiner dreikantigen Ecke geschnitten war. Das Mineral erscheint in dem ziemlich dicken Schliffe lichtrötblich durchsichtig, und es zeigen im gewöhnlichen Lichte weder regelmassig angeordnete Einschlusse, noch sonstige Erscheinungen eine Feldertheilung an, welche im polarisirten mit grosser Deutlichkeit zum Vorschein kommt. Die Platte besteht aus sechs oplisch ziemlich stark wirksamen Theilen, zwischen welchen optisch isotrope uberhaupt 
nicht beobachtet werden konnten, und von deren Anordnung untenstehendes Schema ein Bild geben soll. Drei alternirende Felder von rhombischer Form weisen auf einen Aufbau aus Anwachspyramiden nach dem Rhombendodekaëder hin, zwischen welchen drei Streifen mit parallelen Grenzen liegen, die auf einen Aufbau nach dem Ikositetraëder $\{211\}$ deuten, eine Form, welche zwar an den meisten Krystallen des Vorkommnisses zu beobachten ist, dem zu dem Schliffe verwendeten aber vollständig fehlte. Alle sechs Felder sind optisch zweiaxig und es liegt in allen die optische Axenebene || zu den Diagonalen des durch den Schnitt gebildeten Sechsecks, in welcher Richtung uberall der schiefe Austritt einer Axe zu beoachten ist. In den rhombischen

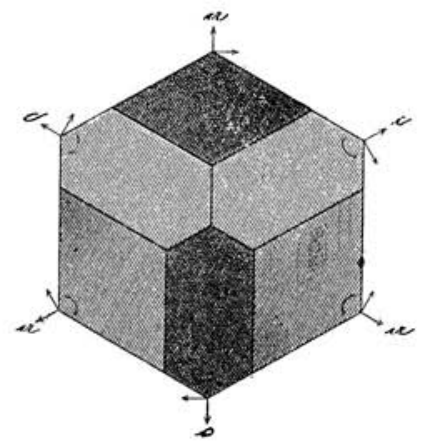
Feldern ist diese Diagonale die Richtung grösster Elasticität, während in denjenigen, welche dem Ikositetraëder entsprechen, die Axe kleinster Elasticităt mit derselben zusammenfällt. Die letzteren Sectoren löschen einheitlich aus und zeigen etwas schwächere Doppelbrechung als die ersteren, in welchen zum Theil die Auslöschung undulös, zum Theil eine deutliche Zwillingslamellirung vorhanden ist, wie sie obige Figur andeutet.

Der hohe Mangangehalt dieses neuen Granatvorkommens lässt einen gewissen Zusammenhang mit den so uberaus manganreichen Pegmatiten des bayerischen Waldes vermuthen, da die sonstigen Mineralien, welche in dem Erzlager selbst vorkommen, dieses Element höchstens in untergeordneter Menge aufweisen.

Was die optischen Eigenschaften anlangt, so lag eine Vergleichung des hier beobachteten Spessartins mit dem Originalspessartin von As chaffenburg nahe, welcher nach der Analyse von Kobell*) eine nicht weit abweichende Zusammensetzung besitzt, in seinem Vorkommen als primärer Gemengtheil des Granits sich aber von demselben unterscheidet. Es ergab sich dabei zunächst, dass die zuletzt besprochenen Krystalle häufig nur aus einem dunnen Netzwerk von fast farbloser Granatsubstanz bestehen, zwischen welchem die ubrigen Gemengtheile des Granils in grosser Menge eingeschlossen sind und sich ferner reichlich dunkelbraune Manganoxyde abgelagert haben, welchen dieser Granat wohl seine makroskopisch dunkelbraunrothe Färbung verdankt. Eine Isolirung reiner Granatsubstanz er-

*) Vergl. Ko bell, Sitzungsber. Münch. Akad. 1868, 2, 292; es dürfte sich daselbst bei der Beschreibung der Trennung des Mangans von den Sesquioxyden ein Druckfehler eingeschlichen haben, da diese Trennung mittelst $\mathrm{NaHCO}_{3}$ nicht auszuführen ist. 
scheint nach dem mikroskopischen Befunde bei diesem Vorkommnisse iusserst schwierig. Wo zusammenhängende Partien von reinem Granat vorhanden waren, liess sich auch bei Anwendung der empfindlichsten Hülfsmittel keine Doppelbrechung constatiren. Die Erscheinung, dass zwei sich in chemischer Beziehung so nahe stehende Granatmineralien in den verschiedenen Formen ihres Auftretens sich optisch so verschieden verhalten, ist sehr auffallend und es ist Analoges bis jetzt noch nicht beobachtet worden. Allerdings sind unter den verschiedenen Gliedern der Granatgruppe nur wenige bekannt, welche sich sowobl als primäre Gemengtheile von Massengesteinen, als auch in Form secundärer Kluftausfullungen finden, allein diese wenigen Beispiele, vor allem die titanhalligen Kalkgranaten, weisen in den verschiedenen Formen ihres Auftretens stets genaue Uebereinstimmung in den optischen Verhältnissen auf. Eine Wiederholung der Analyse des Aschaffenburger: Spessarlins erscheint übrigens schon deshalb nothwendig, weil die Krystalle dieses Vorkommnisses selbst mit unseren modernen Milteln nur schwierig so weit zu reinigen sind, dass man daraus fur eine chemische Analyse nur einigermassen einwandfreies Material gewinnen kann.

Was das dritte neue Vorkommniss dieser Erzlagerstälte, den Turmalin, betrift, welcher am "Rothen Koth « bei $Z$ wiesel in dem dortigen erzfübrenden Granatgneiss aufgefunden wurde, so bildet dieser ein Nest schwarzer prismatischer Krystalle ohne Endausbildung, welche im Dunnschliffe blau durchsichtig erscheinen und die gewöhnliche kräftige Absorption aufweisen. Erwähnt soll noch werden, dass sich unter den neuen Funden aus der Bodenmaiser Erzlagerstätte auch der Desmin wieder befindet, welcher in zahlreichen Krystallen der gewöhnlichsten Combination auf dem Erz und dem umgebenden Cordieritgneiss aufgewachsen erscheint.

Wenn man die neuen Mineralien und im Zusammenhange damit die Art des Vorkommens des Erzes in linsenförmigen Anreicherungen im Cordierilgneiss zunächst am Contact mit dem Granit betrachtet, welche mit der Entfernung vom Granit auch an Mächtigkeit abnehmen, so sprechen vor allem Andalusit und Turmalin für die Auffassung dieser Lagerstätte als einer Contactlagerstätte, während das Vorkommen des so manganreichen Spessartins mit den ubrigen manganreichen Nachschuben der granitischen Intrusion, welche allenthalben in der Umgebung in Form von Pegmatiten zu beobachten sind, in Zusammenhang gebracht werden muss.

Anhangsweise mögen noch zwei Mineralvorkommnisse erwähnt werden, welche in den Pegmatiten der Umgebung von Bodenmais-Zwiesel aufgefunden wurden und von welchen das eine, ein zersetzter Mangan apatit von der Frat bei Bodenmais, wieder einen Beweis fur den ungewöhnlichen Mangangehalt dieser Vorkommnisse bietet. Derselbe fand sich in einem durch $\infty P$ und $O P$ begrenzten Krystalle aufgewachsen auf einer 
Druse des Pegmatits. Der Krystall erscheint oberflächlich gritnlichweiss und trübe und ist aus drei Schalen aufgebaut, von welchen die ausserste, farblose etwa $\frac{1}{4} \mathrm{~mm}$ dick ist und aus normalem Apatit besteht, während darunter eine doppelt so starke Lage von dunkelbrauner Farbe folgt, in welcher man unter dem Mikroskope massenhaft und zumeist \| der c-Axe in farblose Apatitsubstanz eingelagert dunkelbraune Substanzen erkennt, welche aus Manganoxyden bestehen und dem Mineral eine merkliche Absorption des ausserordentlichen Strahles ertheilen. Der innerste Kern des Krystalles endlich, $3 \frac{1}{2} \mathrm{~mm}$ im Durchmesser, ist lichter brauu und unterscheidet sich von der daruber befindlichen Zone nur durch die bedeutend geringere Menge der braunen Einschlüsse. Da die drei Zonen durch scharfe Grenzen von einander geschieden sind, durfte es sich wohl um einen zersetzten Manganapatit handeln, welcher einen zonaren Aufbau aus Schichten von verschiedenem Mangangehalt besass, wobei das leichter zerselzliche Manganphosphat zuerst der Einwirkung der Atmosphärilien erlag. Das Mineral stellt ein Analogon zu dem von Sandberger*) aus einem Pegmatit bei Zwiesel beschriebenen Manganapatit dar.

Die Art und Weise, wie sich namentlich die dunkelbraune Schicht unter dem Mikroskope verhält, erinnert an das Vorkommen von Apatit in einer Anzahl von Eruptivgesteinen, in welchen das Mineral ähnlich wie hier zur $c$-Axe eingelagerte braun durchsichtige, langgestreckte Substanzen beherbergt, wobei gleichzeitig eine kräftige Absorption des ausserordentlichen Strahles auftritt. Es dürfte sich bei einzelnen gleichfalls um einen manganhaltigen Apatit handeln; nachgewiesen wurde dies direct auf mikrochemischem Wege nach Behrens in dem pleochroitischen Apatit des Isenits vom Sengelberge bei Salz.

Das letzte der hier zu erwähnenden neuen Mineralvorkommnisse bil-, det der Kakoxen aus dem bekannten mineralreichen Quarzbruche am Huhnerkobel bei Rabenstein unweit Zwiesel. In einigen der bekannten Triphylinpseudomorphosen fanden sich neben anderen Eisen- und Manganphosphaten radialfaserige, strohgelbe bis gelblichbraune Nadelaggregate mit schwachem Seidenglanz, welche Hohlräume auskleiden. Die mikrochemische Untersuchung ergab, dass dieselben Eisenoxyd, Phosphorsäure und Wasser enthalten. Auch die optische Untersuchung zeigte volle Uebereinstimmung mit den Bestimmungen am Kakoxen, welche von H. Fischer ${ }^{* *}$ ) ausgefuhrt wurden, indem die Axe kleinster Elasticität einen Winkel von etwa $8^{0}$ mit der Längsaxe der Nadeln bildet, und die

*) S a n d berger, Neues Jahrb. Mineral. 1885, 1, 171.

**) Der hier untersuchte Kakoxen zeigt die von H. Fis ch er angegebene Auslöschungsschiefe, ebenso wie eine Anzahl anderer Vorkommnisse, welche bei dieser Gelegenheit geprüft wurden; die Angabe von L. Lu quer, dass der Kakoxen gerade auslöscht lässt sich somit nicht aufrecht erhalten. 
364 E. Weinschenk. Ueber einige neue Mincralvorkommen des bayer. Waldes.

Krystalle, welche lichtgelb durchsichtig erscheinen, parallel zu dieser Richtung etwas slärker absorbiren. Dieses Mineral ist bisher als Zersetzungsproduct des Triphylins nicht bekannt geworden. Ueberhaupt sind hier eine grössere Anzahl nicht genauer untersuchter, wasserhaltiger Phosphate vorbanden, auf welche zurückzukommen sich vielleicht später Gelegenheit findet.

Zum Schlusse gestatte ich mir noch, dem Conservator der königl. bayerischen mineralogischen Staatssammlung, Herrn Prof. Groth, fur die liebenswurdige Ueberlassung des werthvollen Materials meinen herzlichsten Dank auszusprechen.

München, August 1895. 